\title{
On the recovery of the stress-free configuration of the human cornea
}

\author{
Andrea Montanino ${ }^{1}$, Anna Pandolfi ${ }^{2}$
}

${ }^{1}$ Dipartimento di Strutture per l'Ingegneria e l'Architettura, Università degli Studi di Napoli "Federico II", Napoli, Italy, ${ }^{2}$ Dipartimento di Ingegneria Civile ed Ambientale, Politecnico di Milano, Milano, Italy

\section{Abstract}

Purpose: The geometries used to conduct numerical simulations of the biomechanics of the human cornea are reconstructed from images of the physiological configuration of the system, which is not in a stress-free state because of the interaction with the surrounding tissues. If the goal of the simulation is a realistic estimation of the mechanical engagement of the system, it is mandatory to obtain a stress-free configuration to which the external actions can be applied.

Methods: Starting from a unique physiological image, the search of the stress-free configuration must be based on methods of inverse analysis. Inverse analysis assumes the knowledge of one or more geometrical configurations and, chosen a material model, obtains the optimal values of the material parameters that provide the numerical configurations closest to the physiological images. Given the multiplicity of available material models, the solution is not unique.

Results: Three exemplary material models are used in this study to demonstrate that the obtained, non-unique, stress-free configuration is indeed strongly dependent on both material model and on material parameters.

Conclusion: The likeliness of recovering the actual stress-free configuration of the human cornea can be improved by using and comparing two or more imaged configurations of the same cornea.

Keywords: human cornea, inverse analysis, parameter identification, postoperative cornea, preoperative cornea, stress-free configuration

Correspondence: Dipartimento di Ingegneria Civile ed Ambientale, Politecnico di Milano, P.za Leonardo da Vinci, 32 - 20133 Milano, Italy.

E-mail: anna.pandolfi@polimi.it 


\section{Introduction}

Among all the soft biological tissues of the human body, the cornea is unique because of the transparency ${ }^{1}$ and the accessibility of its location. These features make the cornea one of the most deeply studied and better known biological materials, since advanced optical imaging has revealed all the details of the underlying microstructure. ${ }^{2,3}$

The cornea belongs to the system of lenses that in the eye deviate the light rays onto the specialized receptor cells of the retina. When the lens system is defective, the image appears blurred and unfocused, requiring the use of additional lenses to perceive a correct image. Spectacles and contact lenses have been and are largely used, but in the last two decades laser technology has allowed to correct refraction errors permanently by modifying the refractive power of cornea, in consideration of the accessibility of the organ. The change of the corneal refractive power is principally obtained by selective laser ablation of some portions of the tissue, ${ }^{4}$ or by the insertion of small prosthesis or devices (arcuates, intraocular lenses, rings, etc.) within the cornea. Refractive surgery technologies have become very safe and precise, but still gross errors are occurring occasionally when the cornea presents geometrical or structural anomalies. For non-standard corneas that must undergo refractive surgery, the support of a numerical model of the cornea with patient-specific features may become of great importance to reduce the possibility of mistakes and to help in the selection and design of the optimal treatment.

A numerical model of the human cornea must be constructed accounting for all the important features of the tissue. Given the refractive function of the cornea, the adoption of the patient-specific shape is a must. Nowadays, the availability of optical apparatuses makes trivial the attainment of the customized shape that can be transferred in the solid model of the cornea. ${ }^{5}$

As a soft biological tissue, the cornea is very deformable and water rich, and therefore, almost incompressible. The mechanical behavior in physiological conditions is characterized by reversibility even at large deformations. Relaxation experiments on pig corneas have revealed a viscous-plastic-damaging behavior. ${ }^{6}$ Nevertheless, in applications concerning refractive surgery, degenerative aspects should not be of relevance, since the postoperative cornea is supposed to behave in a reversible way as in the preoperative conditions. Thus, in the literature it has become customary to adopt finite strain hyperelastic models for the material, which imply directly reversibility. ${ }^{7,8}$

Although the corneal tissue is organized in five layers, from the mechanical point of view the most important properties are related to collagen, the structural component of the stroma, the central and thicker layer. Stromal collagen, immersed in a matrix of elastin and proteoglycans, is organized hierarchically in fibrils and lamellae following a complex architecture that has been observed more than three decades ago. ${ }^{2}$ In the central area of the cornea the lamellae are preferentially oriented in two directions: nasal-temporal ( $\mathrm{NI}$ ) and superior-inferior (SI). This organization involves approximately $60 \%$ of the fibrils, while the remaining $40 \%$ are randomly oriented. 
The change in curvature in the limbus zone is related to the presence of a consistent amount of fibrils aligned in the circumferential direction. ${ }^{9}$ The distribution of the fibrils is not homogeneous across the corneal thickness. Biomedical imaging has revealed recently that collagen lamellae in the posterior cornea are commonly twice as thick as those in the anterior ${ }^{10}$ and interlamellar interaction results from interweaving, ${ }^{11}$ leading to a shear stiffness three times larger than the one in the posterior third of the stroma. ${ }^{12}$ At the limbus, the larger stiffness is shown at the posterior side, where the limbus merges with the iris. ${ }^{13}$ The architecture of the cornea confers obvious characteristics of inhomogeneity and anisotropy to the material. Thus, the material response varies with the position on the mid-surface, with the position across the thickness of the cornea, and with the direction of loading, and these features are very important in establishing the optimal state under physiological conditions. The optimal state must be intended from the energetic point of view, as a configuration characterized by a minimum of the mechanical energy of the system, where the stresses are balancing the external actions. In particular, the cornea is stressed by the intraocular pressure (IOP) exerted by the aqueous humor that fills the anterior chamber of the eye.

The configuration of the cornea taken by optical imaging (physiological configuration) is stressed, and as such, the geometrical models of the cornea obtained by imaging cannot be directly used in numerical applications. Models require to be integrated by accounting for the unknown physiological stress state in the imaged configuration (pre-stress approach; ${ }^{7}$ ) or by detecting a stress free geometrical configuration to which the IOP is applied. ${ }^{8,14}$ In fact, if the imaged geometry is used directly, the application of the IOP on the posterior surface of the cornea will modify ostensibly the configuration of the cornea, changing the refractive power and the stress state. As in other biological cases, a correct modeling of the cornea requires to recover the stressfree configuration (also known as natural configuration) to which the external actions are applied. The configuration reached by the system under the proper actions will coincide, in this way, to the physiological configuration. The procedure used to recover the stress-free state of a system can be named identification of the natural configuration.

The importance of the recovery of the stress-free geometry in human arteries has been pointed out in Raghavan et al., ${ }^{15}$ where an iterative procedure based on the observation of the self-similarity of the shape of abdominal aortic aneurisms under different blood pressures was used. More recently, a backward displacement method able to solve the inverse problem iteratively using fixed point iterations was described by Bols et al. ${ }^{16,17}$ The correct estimate of the physiological stress state is an important task for arterial walls, loaded by the blood pressure. Disregarding or approximating in a rough way the physiological stress invalidates the predictions on aneurysm formations and vessel ruptures. ${ }^{18-20}$ The importance of the prestress in scleral shells has been pointed out also by Grytz and Downs, ${ }^{14}$ who developed a Forward Incremental Prestressing Method for the computation of the prestress in the physiological configuration. 
As far as the cornea is concerned, the need to recover the stress-free state has been considered in several contributions. An approach based on iterative estimation of the physiological stress was proposed in Pinsky et al. ${ }^{7}$ A method based on the modification of the coordinates of the discretized model has been proposed in Pandolfi and Manganiello, ${ }^{8}$ and the same concept has been applied in subsequent works. ${ }^{21-23}$ A similar procedure has been used by Ariza-Gracia's group. ${ }^{24-27} \mathrm{~A}$ variational approach based on iterative finite element solutions was proposed in a study by Otani and Tanaka. ${ }^{28}$

The approaches described in the literature have a comparable validity, as long as they are able to reconstruct the physiological state in terms of stresses and strains. What has not been sufficiently emphasized is that the stress-free configuration is dependent on the chosen material model and on the values of the material parameters. Identification procedures have been used in combination with Mooney-Rivlin material models, ${ }^{29}$ Ogden material models, ${ }^{30}$ neo-Hookean material models, ${ }^{31}$ Yeoh material models, ${ }^{27}$ or with more realistic fiber reinforced models. ${ }^{8,22,23,32}$ Clearly, the predicted stress state in physiological conditions will be very different in all these cases.

The dependence on material model and parameters renders the identification of the stress-free geometry very delicate and not definitive. The consequence of this uncertainty is that, for a chosen material model, the identification of the stress-free geometry cannot be disjointed from the simultaneous identification of the material parameters. This means that a single configuration, or a single image, is not sufficient to characterize at once geometry and materials, calling for the need of conducting invivo tests on each patient. ${ }^{26,33}$ Furthermore, no useful information of the in-vivo mechanical properties can be derived from ex-vivo tests, ${ }^{34}$ which deal with a completely different material removed from its natural environment. Possible candidates for the use in identification procedures are the probe test ${ }^{35}$ and the air puff test. ${ }^{25,26,36-39}$

As an alterative, when two images corresponding to two different configurations of the same cornea are available, it is possible to characterize a reduced selection of the material parameters together with the stress-free configuration. This can be possible, for example, when images of the same cornea in preoperative and postoperative conditions are available. The approach has been used in a rather successful way in several works using anisotropic material models with inclusion of the fibril microstructure and considering preoperative and postoperative geometries of corneas that underwent photo-refractive keratectomy (PRK), 5,32,36,37,40 but an accurate analysis on the influence of the material model on the identified material properties and stress-free geometry has never been conducted.

Goal of this study is to gain awareness on the relevance on the choice of a material model in the identification of the stress-free configuration and of the material parameters.

Therefore, we consider a set of patient-specific corneas that underwent laser reprofiling surgery (PRK) and three material models characterized by a growing complexity: the isotropic Hooke material model extended to the finite kinematics, the Mooney-Rivlin material model, and the sophisticated anisotropic model proposed in 
Pandolfi and Vasta. ${ }^{23}$ We describe the approach for the simultaneous identification of the natural configuration and a limited number of material properties of the adopted material models, present the results obtained on the set of patient specific corneas, and discuss the numerical findings in view of possible applications.

\section{Methods}

The procedure of the identification of the stress-free geometry and of a selected number of material parameters based on the comparison of the preoperative and postoperative physiological configurations of a PRK-ablated cornea has been conceived on the following idea. ${ }^{40}$

Cornea reprofiling conducted with laser ablation removes the anterior tissues of the cornea, including epithelium, Bowman's membrane and a certain amount of the anterior stromal tissue. Clearly, ablation burns the tissue, and causes temporary modifications in the immediately adjacent tissues that need a week or more to heal and renew the epithelial layer. Since the posterior surface of the cornea is not directly touched by the laser, it is possible to make the assumption that the posterior third of the cornea is not affected. An additional assumption is that IOP is not modified by refractive surgery.

The stress-free configuration is intended as an ideal non-physiological state where the material is not loaded, or the IOP is zero. The stress-free state is not known, and cannot be achieved under in vivo conditions, but it can be estimated through an inverse numerical calculation. Having chosen a material model and a set of material parameters, a numerical analysis where the posterior surface of the cornea is pressurized with the physiological IOP will provide, as solution, the displacement field associated to the stresses that balance the IOP by means of the material model. The displacements modify the cornal configuration, which will not longer respect the physiological shape.

Let us now assume to subtract the computed displacements to the original coordinates of the cornea in the physiological configuration. The resulting configuration will be less convex than the physiological configuration. If a new analysis under the same IOP is conducted on the modified geometry, the resulting stressed geometry will be closer to the physiological geometry. Clearly, there will be differences related to the fact that the analysis has to be conducted in finite kinematics, which induces geometrical non-linearity; therefore the procedure must be repeated several times using an iterative algorithm that will be interrupted when the desired precision is reached.

The same procedure can be applied to the preoperative and postoperative configurations of the corneas, using the same material model and parameters. If the choice of material model and parameters are correct, the posterior surfaces of the stressfree geometries of the two cases will coincide. If they do not, the material model, the material parameters, or both are not correct.

The discrepancy between the coordinates of the posterior surfaces of the preop- 


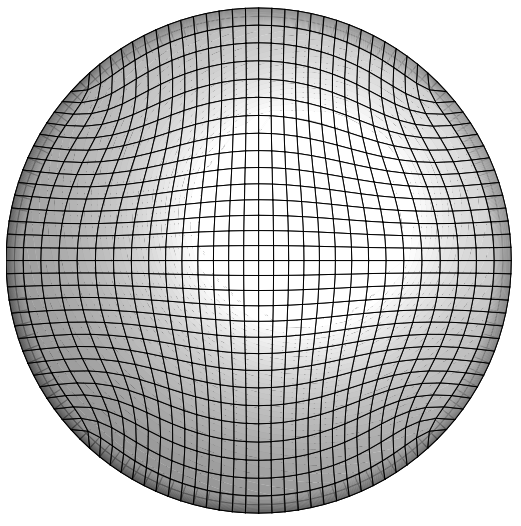

(a) Top view

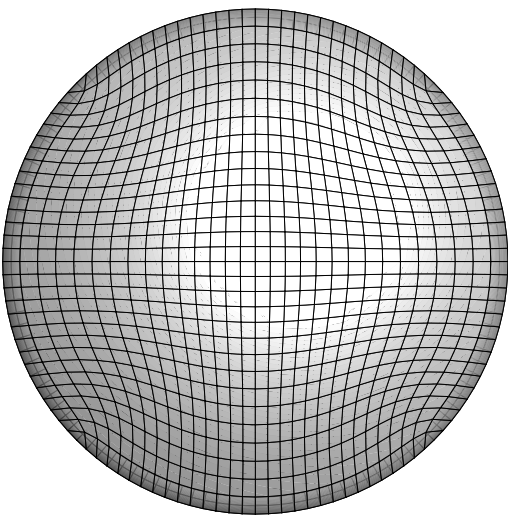

(b) Bottom view

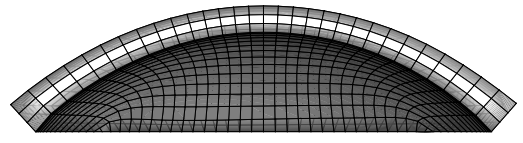

(c) Section

Fig. 1. Example of finite element discretization for one of the corneas considered in this study. The geometry refers to the physiological preoperative configuration.

erative and postoperative stress-free geometries can be taken as a measure of the likeness (ML) of the material parameters for the adopted material model. The ML can then be used to identify the optimal set of material parameters for the patient-specific cornea.

The actual procedure is described in detail in the following section.

\subsection{Recovery of the stress-free configuration of the cornea}

Twelve pairs of preoperative and postoperative corneal geometries were chosen in a random way from a large set of informed patients that underwent PRK refractive surgery. The data used in this work were collected by the same experienced surgeon using a high definition corneal tomographer coupled with a pachymeter, according to a protocol approved by the Italian Data Protection Authority and to the principles expressed in the Declaration of Helsinki. Purely geometrical data elaborated from the images by the tomographer were anonymized and de-identified prior to the transmission to us and disjoined from all the other clinical information (age, gender, ethnicity, and IOP).

Data are provided as a list of coordinate of a cloud of points representing the anterior and posterior surface of the cornea in preoperative or postoperative configu- 
ration. The data are elaborated through a software to produce a solid model of the cornea discretized in finite elements, as shown in Figure 1.

To each cornea, we apply the procedure proposed firstly in Pandolfi and Manganiello. ${ }^{8}$ The algorithm of identification of the stress-free geometry begins with the construction of the mesh in the physiological configuration, seen as a final target of the iterative process. Each step consists in the application on the posterior surface of the cornea of the patient-specific IOP, keeping the limbus constrained to rotate in order to maintain the cross-section always orthogonal to the deformed mid-surface of the cornea. The rotating boundaries have been proved to be the ones that optimize the refractive behavior of the cornea in the physiological IOP range..$^{21}$ The solution of the static problem with an assigned material model provides the set of displacements employed in the iterative algorithm to compute the stress-free configuration. The iterative process ends when, within an assigned tolerance $\epsilon$, the deformed configuration superposes the target physiological configuration. The algorithm is described in Algorithm. 1

Algorithm 1 Unstressed geometry recovery algorithm, as proposed in Pandolfi and Manganiello ${ }^{8}$

1: Set $\mathbf{X}_{0}$ as the nodal coordinates corresponding to the physiological IOP.

2: Set $k=1$

3: Set $\mathbf{X}^{k}=\mathbf{X}_{0}$

4: for $k \leq k_{\max }$ do

5: $\quad$ Assign the physiological IOP on the posterior surface of the cornea

6: $\quad$ Solve the static problem and obtain the $k$-th nodal displacements $\mathbf{u}^{k}$

7: $\quad$ Compute the $k$-th physiological nodal coordinates $\mathbf{x}^{k}=\mathbf{X}^{k}+\mathbf{u}^{k}$

8: $\quad$ Compute the coordinate difference $\Delta \mathbf{X}=\mathbf{X}_{0}-\mathbf{x}^{k}$ and the corresponding norm $|\Delta \mathbf{X}|$

9: if $|\Delta \mathbf{X}|<\epsilon\left|\mathbf{X}^{0}\right|$ then

10: $\quad$ Exit

11: end if

12: $\quad$ Set $\mathbf{X}^{k+1}=\mathbf{X}_{0}-\mathbf{u}^{k}$

13: $\quad$ Set $k=k+1$

14: end for

\subsection{Identification of the optimal material properties}

For the chosen material model, the identification of the optimal material properties is achieved by comparing the coordinates of the nodes lying on the posterior surface of the same cornea in the preoperative and postoperative configurations. In the following, the set of $p$ material properties is referred to as $\mathbf{c}=\left\{c_{2}, c_{2}, \ldots, c_{p}\right\}$. Note that, in general, the material property set will include material parameters, parameters related to inhomogeneity and anisotropy distribution, variability across the thickness, 
and others. Moreover, the set might not include all the parameters of the chosen material model; when a parameter is sufficiently well characterized by other means, it might be excluded from the $\mathrm{c}$ set.

The recovery procedure described in the previous section is applied to both the preoperative and postoperative configuration, using the same material properties and the same IOP, leading to two sets of nodal coordinates, $\mathbf{X}^{\text {pre }}$ and $\mathbf{X}^{\text {post }}$, respectively. Both sets will be dependent on the material properties.

Given the obvious difference in the geometry due to the cornea reprofiling, the comparison between stress-free preoperative and postoperative configuration can be conducted only on the nodes lying on the posterior surface of the cornea. The coordinates of the nodes of the posterior surface are collected in the subsets $\mathbf{Y}^{\mathrm{pre}}$ and $\mathbf{Y}^{\text {post }}$, respectively.

We introduce the index $M L$ representing a measure of likeness of the property set c as

$$
\operatorname{ML}(\mathbf{c})=\frac{\left|\mathbf{Y}^{\text {post }}-\mathbf{Y}^{\text {pre }}\right|}{\left|\mathbf{Y}^{\text {pre }}\right|}
$$

where the norm $|\mathbf{X}|$ is defined as

$$
|\mathbf{X}|=\sqrt{\sum_{1}^{N}\left(X_{1}^{2}+X_{2}^{2}+X_{3}^{2}\right)}
$$

and $N$ is the number of elements in the subsets $\mathbf{X}$. Clearly, the more likely the material properties are, the smaller is the value of the ML index. Thus, the identification procedure can be stated as an optimization problem

$$
\mathbf{c}^{\mathrm{opt}}=\arg \min _{\mathbf{c}} \mathrm{ML}(\mathbf{c}) \text {. }
$$

In this work, the search for the optimal values of the parameters is organized by spanning discrete values of the $p$ parameters within a realistic range, determined through a few preliminary calculations. For each set of values of $p$, we apply the iterative recovery procedure for both preoperative and postoperative cases and compute the ML index. Since each evaluation of ML involves a certain number of finite element analyses, the efficiency of the approach is clearly related to the chosen material model, which may affect considerably the computational time requested by the static solution.

In the following calculations, the missing value of the patient-specific IOP is assumed to be equal to $14 \mathrm{mmHg}(1.87 \mathrm{kPa})$ for all cases.

\section{Results}

The method is applied to three different material models. The first material model is a Hooke material extended to the finite kinematics. The second material model 
is a Mooney-Rivlin model. The third material model is the second order approximation anisotropic model, accounting for the complex architecture of distributed collagen fibrils within the cornea, described in Pandolfi and Vasta ${ }^{23}$ and used in previously cited studies. ${ }^{5,32}$.

\subsection{Hooke material model extended to finite kinematics}

The material model considered here is a fictitious material, since it adopts the Hooke model to finite kinematic stress and strain measure. The model can be expressed through the strain energy density

$$
\Psi_{\text {Hooke }}=\frac{1}{2} \mathbf{E}: \mathbb{D}(E, \nu) \mathbf{E}, \quad \mathbf{S}=\frac{\partial \Psi_{\text {Hooke }}}{\partial \mathbf{E}}
$$

where $\mathbf{E}$ is the Green-Lagrange strain tensor, $\mathbf{S}$ the second Piola-Kirchhoff tensor, and $\mathbb{D}$ is the isotropic constant constitutive tensor, dependent on the Young's modulus $E$ and on the Poisson's coefficient $\nu$. We assume that Poisson's coefficient is known, and set it to $\nu=0.45$, thus the parameter drops from the set $\mathbf{c}$. The optimization problem in Eq. (3) reduces to

$$
E^{\mathrm{opt}}=\arg \min _{E} \mathrm{ML}(E)
$$

Table 1. Geometrical characteristics of the baseline cornea.

\begin{tabular}{|l|c|}
\hline Geometrical parameter & $\mathbf{m m}$ \\
\hline Average anterior surface in-plane radius (AAR) & 5.8100 \\
Average posterior surface in-plane radius (APR) & 5.2900 \\
Pre-operative central corneal thickness (BCCT) & 0.5117 \\
Post-operative central corneal thickness (ACCT) & 0.4414 \\
Maximum ablation profile depth $(\Delta \mathrm{CCT})$ & 0.0720 \\
\hline
\end{tabular}

The approach is described in detail with reference to one of the patient-specific corneas (heretofore referred to as baseline analysis), characterized by the geometrical parameters listed in Table 1 . We begin by constructing a finite element mesh from the supplied tomographer data. The mesh, shown in Figure 1, consists of 2,700 exahedron solid elements, with 30 elements along the NT and SI meridians, and 3 elements across the thickness.

Figure 2 compares the physiological preoperative and postoperative configurations of the NT meridional section of the baseline cornea, as obtained from the tomographer images, showing the whole section Fig. 2(a), and a detail of the optical zone at the center, Fig. 2(b). Images visualize clearly the reshaping induced by the PRK reprofiling and, moreover, testify the forward deflection of the posterior surface induced by the reduction in corneal stiffness.

The optimal value of Young's modulus is obtained by running several recovery analyses with the preoperative and postoperative geometries, assuming for $E$ the discrete values $0.25,0.375,0.5,0.625,0.75,1$, and $1.25 \mathrm{MPa}$. The dependence of the ML 


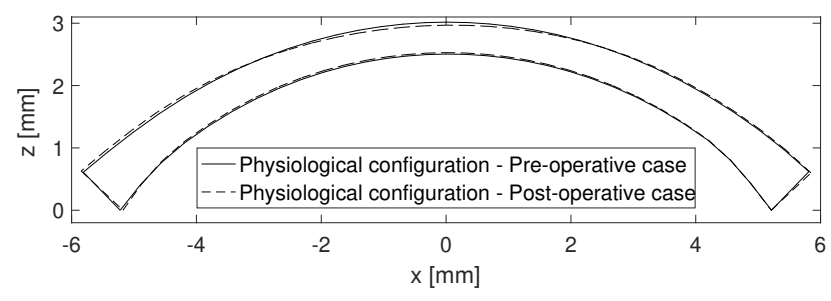

(a) Whole

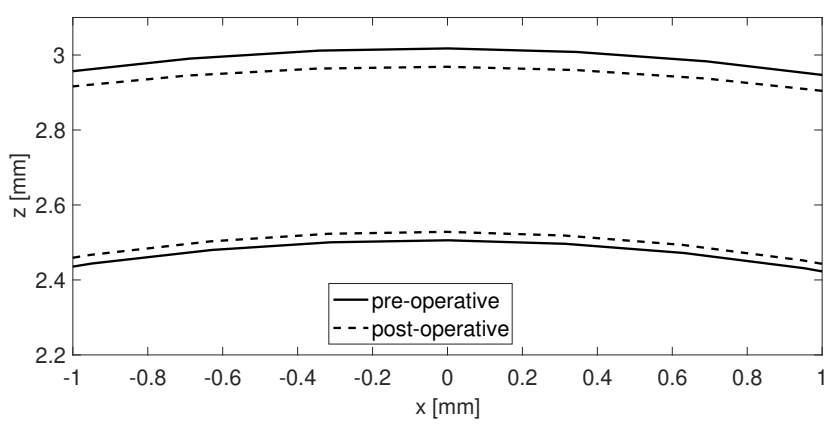

(b) Central Detail

Fig. 2. Baseline cornea, Hooke material. Physiological preoperative and postoperative configurations of the cornea across the NT meridian, as provided by the tomographer. (a) Whole extension. (b) Detail in the central optical zone.

index on $E$ is shown in Figure 3. The minimum value of the ML index, corresponding to $2.546 \times 10^{-5}$ is located at $E^{\mathrm{opt}}=0.625 \mathrm{MPa}$. Figure 4 compares the recovered stressfree preoperative and postoperative geometries corresponding to the optimal value $E^{\mathrm{opt}}$, showing the good correspondence of the position of the posterior surfaces.

The role played by Young's modulus on the recovered stress-free geometry is shown with the aid of Figure 5. Starting from the same physiological postoperative configuration and assuming two different values for Young's modulus, $0.25 \mathrm{MPa}$ and 1.25 MPa, respectively, the recovered geometries are ostensibly different.

The analysis described for the baseline cornea was repeated for 12 pairs of corneal geometries, whose geometrical parameters are listed in Table 2. The baseline cornea is labelled 1S. The same table also lists the minimum value of the $\mathrm{ML}^{\mathrm{opt}}$ index and the corresponding $E^{\mathrm{opt}}$. 
Table 2. Geometrical characteristics of the 12 human corneas used in this study. AAR: average anterior radius

\begin{tabular}{|c|c|c|c|c|c|c|c|}
\hline Cornea & $\begin{array}{c}\text { AAR } \\
\mathrm{mm}\end{array}$ & $\begin{array}{c}\text { APR } \\
\mathrm{mm}\end{array}$ & $\begin{array}{c}\mathrm{BCCT} \\
\mathrm{mm}\end{array}$ & $\begin{array}{c}\text { ACCT } \\
\mathrm{mm}\end{array}$ & $\begin{array}{c}\Delta \mathrm{CCT} \\
\mathrm{mm}\end{array}$ & $\begin{array}{c}E^{\mathrm{opt}} \\
\mathrm{MPa}\end{array}$ & $\mathrm{ML}^{\mathrm{opt}}$ \\
\hline 1S & 5.812 & 5.29 & 0.512 & 0.440 & 0.072 & 0.625 & $2.54610^{-5}$ \\
1D & 5.820 & 5.19 & 0.506 & 0.449 & 0.057 & 0.750 & $3.30410^{-6}$ \\
2S & 5.570 & 5.00 & 0.540 & 0.425 & 0.115 & 0.750 & $3.41510^{-5}$ \\
2D & 5.529 & 4.96 & 0.535 & 0.403 & 0.132 & 1.000 & $1.67210^{-5}$ \\
3S & 5.579 & 5.00 & 0.501 & 0.462 & 0.039 & 0.500 & $3.18010^{-5}$ \\
3D & 5.529 & 5.02 & 0.509 & 0.471 & 0.038 & 0.500 & $2.11610^{-5}$ \\
4S & 5.525 & 5.01 & 0.567 & 0.502 & 0.065 & $<0.125$ & - \\
4D & 5.538 & 5.03 & 0.564 & 0.473 & 0.091 & 0.125 & $3.02010^{-4}$ \\
5S & 5.786 & 5.29 & 0.523 & 0.458 & 0.065 & $<0.125$ & - \\
5D & 5.873 & 5.32 & 0.530 & 0.452 & 0.078 & - & - \\
6S & 5.507 & 4.98 & 0.597 & 0.436 & 0.161 & $<0.125$ & - \\
6D & 5.590 & 5.04 & 0.553 & 0.438 & 0.115 & $<0.125$ & - \\
\hline Average & 5.622 & 5.076 & 0.539 & 0.452 & 0.087 & 0.413 & - \\
Std dev & 0.134 & 0.131 & 0.028 & 0.024 & 0.036 & 0.308 & - \\
\hline
\end{tabular}

APR: average posterior radius. BCCT: Preoperative central corneal thickness. ACCT: post-operative central corneal thickness. $\triangle C C T$ : central ablation depth. $E^{\text {opt }}$ : optimal value of the Young's modulus. $\mathrm{ML}^{\mathrm{opt}}$ : minimum value of the $\mathrm{ML}$ 


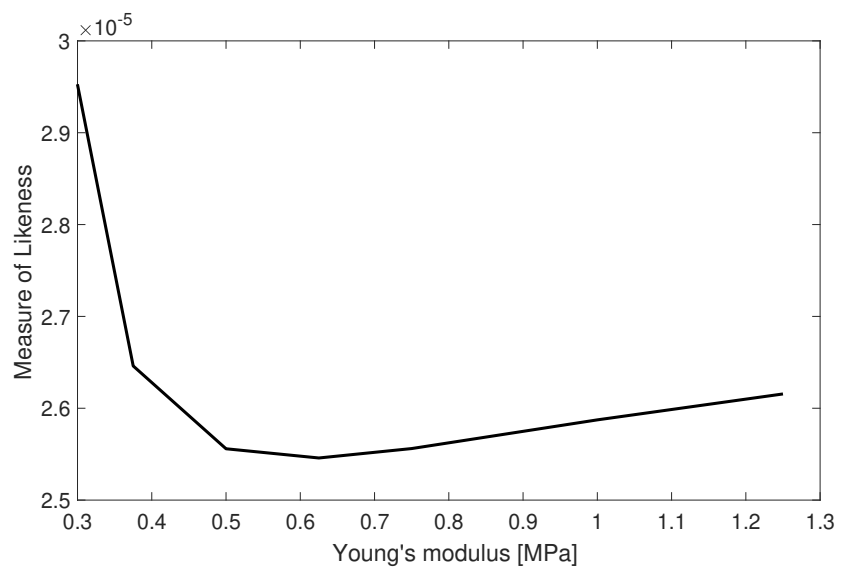

Fig. 3. Baseline cornea with Hooke material model. Dependence of the ML index on the Young's modulus.

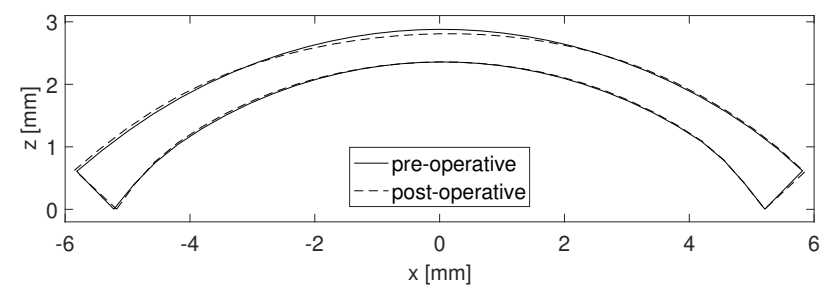

Fig. 4. Baseline cornea with Hooke material model. Comparison between the recovered stressfree preoperative and postoperative geometries for the optimal value of Young's modulus, $E^{\text {opt }}=0.625 \mathrm{MPa}$.

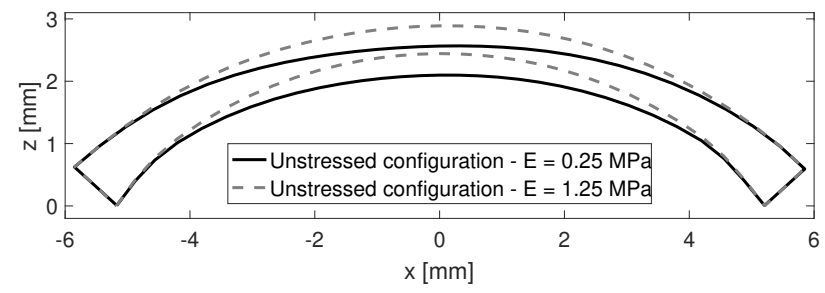

Fig. 5. Baseline cornea with Hooke material model. Comparison between the recovered stressfree preoperative geometries obtained with two different values of Young's modulus $E$, i. e., $0.25 \mathrm{MPa}$ and $1.25 \mathrm{MPa}$, respectively. 
Table 2 shows that, within the explored range $E=\{0.25,1.25\} \mathrm{MPa}$, the corneas labelled 1S, 1D, 2S, 2D, 3S, and 3D are characterized by a minimum value of the ML index, and the corresponding $E^{\mathrm{opt}}$ falls in the range $\{0.5,1\} \mathrm{MPa}$. Regrettably a minimum was not detected for the corneas labelled 4S, 4D, 5D, 5S, 6D, and 6S. Corneas labelled $4 \mathrm{~S}, 4 \mathrm{D}, 5 \mathrm{~S}, 6 \mathrm{~S}$, and $6 \mathrm{D}$ reveal an increasing trend, and the minimum $\mathrm{ML}$ index might fall below $E=0.25 \mathrm{MPa}$. The cornea labelled 5D, instead, shows a decreasing trend that may be ascribed to an imprecise detection of the postoperative image that does not allow alignment to the preoperative image.

To investigate the behavior of the corneas that failed to show a minimum in the range $E=\{0.25,1.25\} \mathrm{MPa}$, for the sole cornea $4 \mathrm{D}$ the analysis has been extended to a wider range exploring the values $E=0.2,0.1125,0.12,0.125,0.13,0.1375$, and $0.15 \mathrm{MPa}$. The second search revealed a minimum of the $\mathrm{ML}$ index for $E=0.125 \mathrm{MPa}$ (Fig. 6). Interestingly, the $M L^{\mathrm{opt}}$ is one order or magnitude larger than in the cases

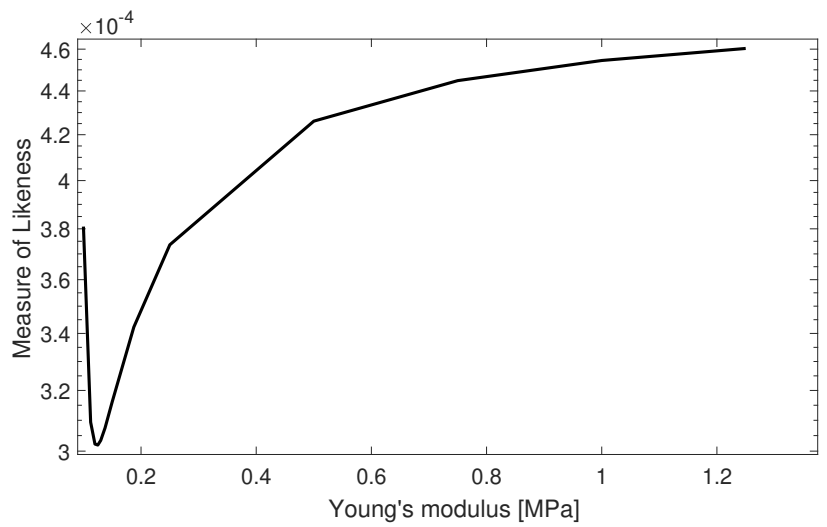

Fig. 6. Anomalous cornea 4D with Hooke material model. Dependence of the ML index on Young's modulus.

that provided a good response in the reduced range of $E$, suggesting that some discrepancy characterizes the data obtained from the preoperative and postoperative images. The discrepancy is indeed confirmed by the comparison of the recovered stress-free preoperative and postoperative geometries, that, for the optimal values of the Young's modulus $E^{\text {opt }}=0.125 \mathrm{MPa}$ does not show any agreement on the shape of the posterior surface (Fig. 7). 


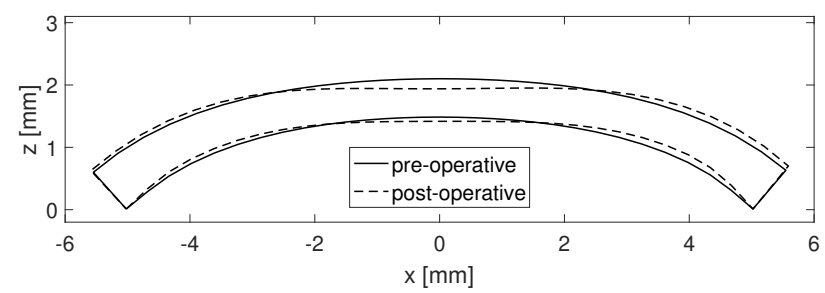

Fig. 7. Anomalous cornea 4D with Hooke material model. Comparison of the recovered stressed-free preoperative and postoperative geometries, at the optimal Young's modulus $E^{\mathrm{opt}}=0.125 \mathrm{MPa}$.

\subsection{Mooney-Rivlin material model}

The quasi-incompressible version of the isotropic Mooney-Rivlin material model is governed by a strain energy function of the form

$$
\Psi_{\mathrm{MR}}=\frac{1}{4} K\left(J^{2}-1-2 \log J\right)+\frac{1}{2} \mu_{1}\left(\bar{I}_{1}-3\right)+\frac{1}{2} \mu_{2}\left(\bar{I}_{2}-3\right)
$$

where $J$ is the determinant of the deformation tensor $\mathbf{F}, \bar{I}_{1}$ and $\bar{I}_{2}$ are the first and second invariant of the isochoric part of the Cauchy-Green strain tensor $\overline{\mathbf{C}}=J^{-2 / 3} \mathbf{F}^{T} \mathbf{F}$, $K$ is a stiffness-like coefficient used to enforce the incompressibility of the material, and $\mu_{1}, \mu_{2}$ are shear stiffness parameters related to the shear modulus of the material as $\mu=\mu_{1}+\mu_{2}$.

In order to reduce the number of parameters to identify to $\mu_{1}$ and $\mu_{2}$, given the meaning of a penalization coefficient for $K$, we set $K=7 \mathrm{MPa}$ in all the calculations.

As already mentioned, in the present work we did not use a true optimization algorithm to detect the optimal values of the parameters, but performed multiple iterative recovery procedures over discrete sets of values. To reduce the heaviness of the calculations in the case of the two-parameter Mooney-Rivlin model, the search for the optimal pair of parameters has been restricted in a rather arbitrary way by performing a two-phase search. In the first phase, we constrain the two parameters to assume the same value and search the optimal value of $\mu_{2}=\mu_{1}$ over discrete values within a wide range. In the second phase, we constrain $\mu_{1}$ to the fixed optimal value and perform a second search on $\mu_{2}$ again over a discrete set of values (Fig. 8). Clearly, since the two searches are discrete and constrained, the algorithm reduces in a sensible way the possibility to find the absolute minimum; thus the present results have to be considered only in a demonstrative way. In particular, we remark that the two parameters cannot be identified in a unique way, since we are comparing only two configurations.

We replicate for the baseline cornea 1D the optimization search conducted for the case of a Hooke material model. The optimization of the parameter $\mu_{1}$ (solid line in 


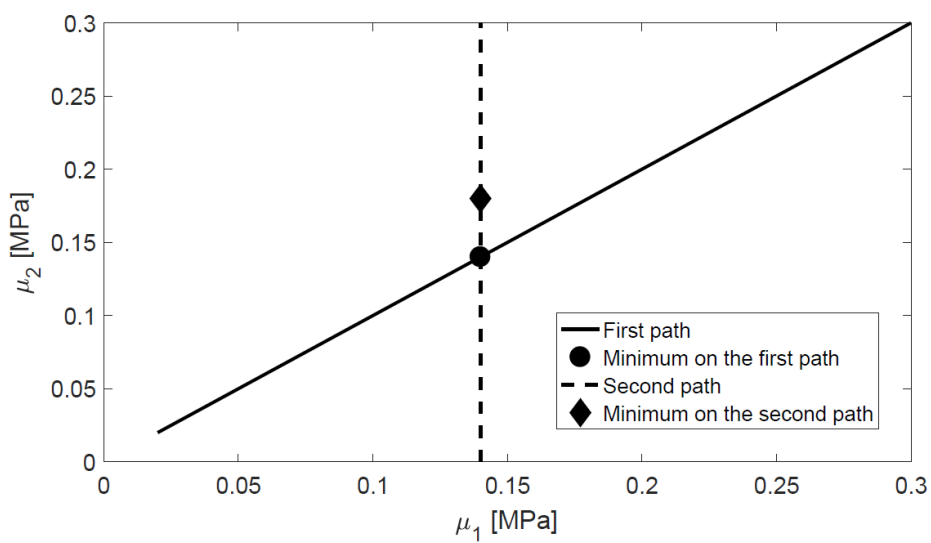

Fig. 8. Visualization of the search strategy adopted for the shear stiffness parameters $\mu_{1}$ and $\mu_{2}$ of the Mooney-Rivlin material model.

Fig. 8) gives $\mu_{1}^{\mathrm{opt}}=\mu_{2}=0.14 \mathrm{MPa}$. The dependence of the ML index on $\mu_{1}$ can be observed in Figure $9(\mathrm{a})$.

Next, the parameter $\mu_{1}^{\text {opt }}=0.14 \mathrm{MPa}$ is kept fixed at the optimal value, and the $\mathrm{ML}$ index is computed for $\mu_{2}$ in the range $\{-0.06,0.30\} \mathrm{MPa}$ (dashed line in Fig. 8). The dependence of the ML index on $\mu_{2}$ is shown in Figure $9(\mathrm{~b})$. The optimal value of $\mu_{2}^{\text {opt }}=0.18 \mathrm{MPa}$ is obtained for a value of $\mathrm{ML}=7.453 \times 10^{-6}$ one order of magnitude inferior to the best value obtained for the Hooke material model. 


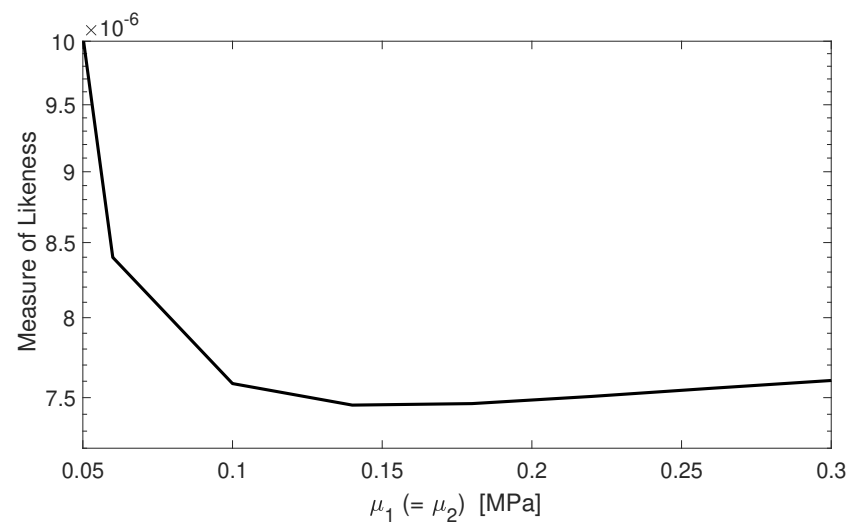

(a) First search

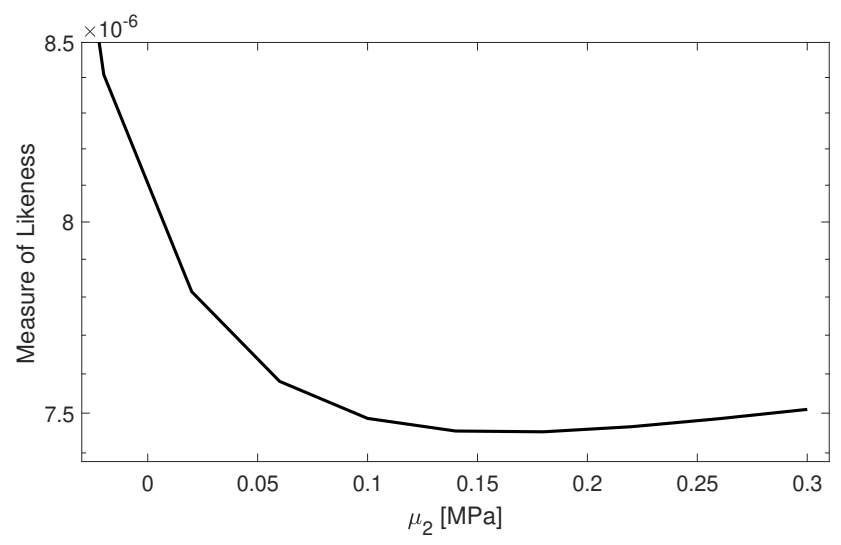

(b) Second search

Fig. 9. Baseline cornea with Mooney-Rivlin material. (a) Dependence of the ML index on the first shear stiffness parameter $\mu_{1}$, under the constraint $\mu_{2}=\mu_{1}$. (b) Dependence of the ML index on the second shear stiffness parameter $\mu_{2}$ for a fixed value $\mu_{1}=0.14 \mathrm{MPa}$.

\subsection{Anisotropic distributed fiber reinforced material model}

The anisotropic distributed fiber reinforced material model here considered is an advanced second order model that accounts for the average and the variance of two distributions of fibers. The model has been developed in Pandolfi and Vasta ${ }^{23}$ and applied successfully to the modelling of the human cornea in several applications. . $^{52,40}$ The model can be thought as an extension of the Mooney-Rivlin material model to account for the anisotropy induced by the presence of dispersed reinforcing fibers 
embedded into an isotropic matrix. The strain energy function has the form

$$
\Psi_{\mathrm{V}}=\Psi_{M R}+\sum_{M=1}^{2} \frac{k_{1 M}}{2 k_{2 M}} \exp \left[k_{2 M}\left(\bar{I}_{4 M}^{*}-1\right)^{2}\right]\left(1+K_{M}^{*} \sigma_{I_{4 M}}^{2}\right)
$$

where $\bar{I}_{4, M}^{*}$ and $\sigma_{I_{4 M}}$ are the average and the variance of the pseudo-invariant measuring the square of the stretch along any spatial direction of the $M$-th set of fibers, $k_{1 M}$ is a stiffness parameter that controls the behavior of the fibers at small strains, $k_{2 M}$ is a dimensionless rigidity parameter that controls the behavior of the fibrils at large strains, and $K_{M}^{*}$ a coefficient dependent on $\bar{I}_{4, M}^{*}, k_{1 M}$, and $k_{2 M}$, cf. $^{23}$

The number of parameters of the material model (seven) makes the search for the non-unique optimal set of parameters rather expensive from the numerical point of view. To illustrate the method, we reduce the problem to the same level of difficulty of the Mooney-Rivlin material model, by assigning the values of the penalty coefficient $K$ and of the four fiber parameters $k_{1 M}, k_{2} M$ according to the data documented in in Sánchez et al. ${ }^{40}$ Therefore, we set $K=5.5 \mathrm{MPa}, k_{11}=k_{12}=0.05 \mathrm{MPa}$, and $k_{21}=$ $k_{22}=200$. Again, the baseline cornea labelled S1 has been used for demonstration. The search is conducted only on the shear stiffness parameters $\mu_{1}$ and $\mu_{2}$, adopting the same strategy described in Section 3.2.

The parameter $\mu_{1}$ has been set as $\{0.04,0.05,0.06,0.07\} \mathrm{MPa}$. The computed ML index as a function of $\mu_{1}=\mu_{2}$ is shown in Figure 10(a). The optimal value of $\mu_{1}^{\text {opt }}=0.05 \mathrm{MPa}$ is clearly shown in the plot, with the corresponding ML index equal to $6.1114 \times 10^{-6}$. The second search is conduced for $\mu_{1}^{\text {opt }}=0.05 \mathrm{MPa}$, varying $\mu_{2}$ in $\{0.03--0.07\} \mathrm{MPa}$. The dependence of the ML index on $\mu_{2}$ is shown in Figure 10(b), and the optimal value is $\mu_{2}^{\mathrm{opt}}=0.05 \mathrm{MPa}$. 


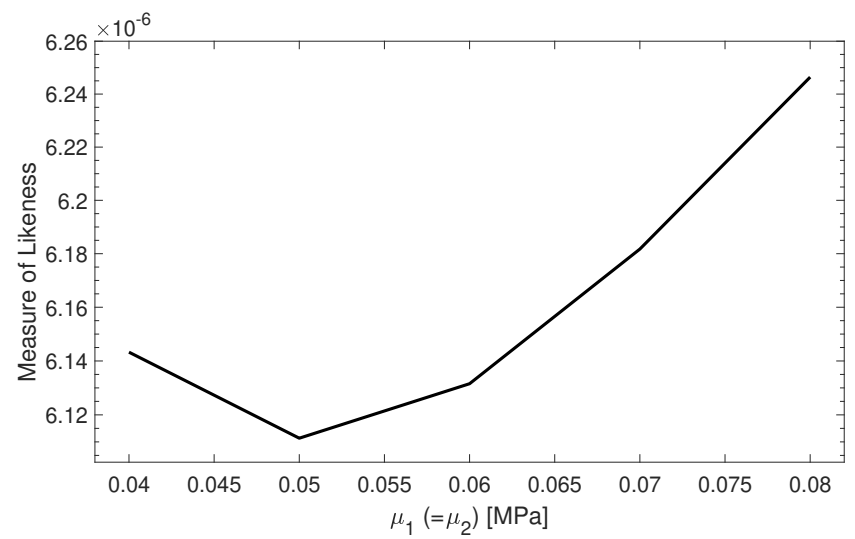

(a) First search

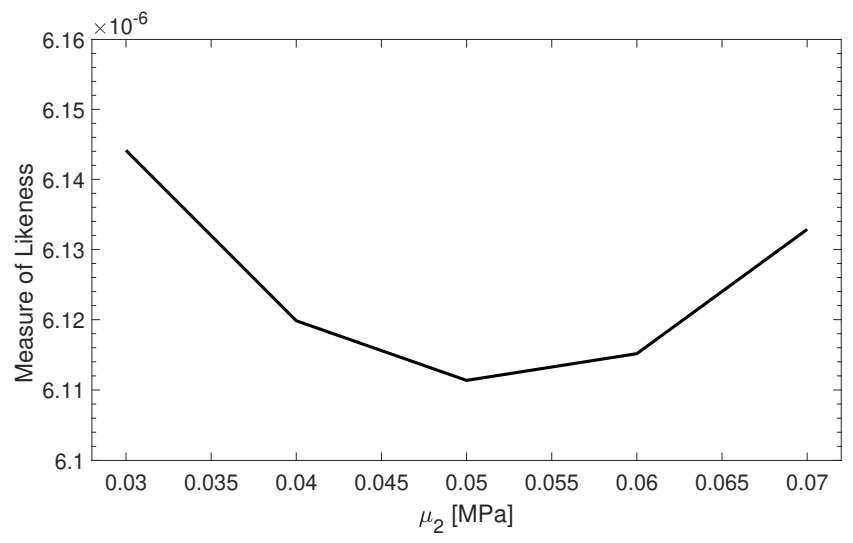

(b) Second search

Fig. 10. Baseline cornea with anisotropic distributed fiber reinforced material. (a) Dependence of the ML index on the first shear stiffness parameter $\mu_{1}$, under the constraint $\mu_{2}=\mu_{1}$. (b) Dependence of the ML index on the second shear stiffness parameter $\mu_{2}$ for a fixed value $\mu_{1}=$ $0.05 \mathrm{MPa}$.

\section{Discussion}

We illustrated a procedure for the simultaneous identification of the stress-free geometry and a reduced set of material parameters for a patient-specific model of a human cornea that underwent laser ablation surgery (PRK). The identification procedure is specifically related to the availability of two comparable configurations, corresponding to a preoperative and a postoperative state, respectively.

The situation is not the ideal one, since the material properties can be identified 
only after the surgery. Therefore the model cannot exploit its predictability in view of optimizing the ablation profile. Nevertheless, we have been motivated by several reasons to conduct this study.

First, the achieved patient-specific model could be employed to estimate the response of the eye to additional surgery, for example, the insertion of an intraocular lens or the execution of a crosslinking procedure. Second, the aim of this work is to describe a conceptually general procedure that can be modified and improved by the suitable selection of two or more comparative configurations of the same cornea, obtained with other means, for example, with a planned protocol of in-vivo tests. Third, the application of this approach to a large set of human corneas that have undergone PRK refractive surgery can be used to restrict the variability of the material parameters of different material models into physically significant ranges of values.

The proposed method must be intended as one of the several ways that allow to identify the material properties of an assigned material model. In particular, the method relies on the existence of an ideal stress-free configuration that cannot be attained under physiological conditions, because it is associated to a non-physiological state, at zero IOP. The ideal unstressed configuration can be determined only through numerical calculations conducted in finite kinematics. In fact, linear elastic approaches are based on the assumption that there is no change of configuration under loading, or no difference between he stress-free and the physiological configuration, excluding the possibility to use the method for the estimation of material parameters. Therefore, the proposed method is suitable only to finite kinematics material models that describe the behavior of deformable soft tissues.

To compensate the missing surrounding tissues, throughout the calculation we adopted special boundary conditions consisting in the adaptive rotation, i. e., a driven motion of the nodes located at the limbus boundary to preserve the planarity of the boundary while enforcing the normality to the mid-surface of the deforming cornea. The concept of rotating boundaries has been presented in a previous study, ${ }^{21}$ as an alternative to fixed boundary (mimicking rigid environment) and to elastic boundaries (mimicking by means of tuned springs the elasticity of surrounding tissues). With respect to other boundary conditions, the adaptive rotations of the limbus have been proven by means of comparative analyses to be the ones providing the most realistic refractive properties of the deforming cornea, showing the minimal variation of the refractive power with growing IOP and the lowest elastic energy stored in the system.

Among the methods proposed in the literature for the identification of the stressfree state, the approach proposed in Grytz and Downs, ${ }^{14}$ where the concept of the comparison between distinct configurations is used to estimate the physiological stress/strain state in biological tissues, stands close to our previously described algorithm. ${ }^{8}$ Although presented in a more formal and elegant way with respect to our work, ${ }^{8}$ the underlying idea is the same: to reconstruct the physiological stress state by searching for a stress-free configuration. The difference between the two methods is that here we are comparing two configurations that differ for tissue mass and volume but withstand the same load, Grytz and Downs ${ }^{14}$ compare two configu- 
rations that differ for loads but preserve mass and volume.

The approach has been illustrated with reference to three material models: the Hooke extended to the non-linear range, the Mooney-Rivlin, and the anisotropic stochastic fiber reinforced model. ${ }^{23}$ The first two material models, which are not particularly suitable for modelling the human corneas, have been considered in order to set up and verify the procedure. In particular, the Hooke model should not be used for soft tissues, since it overestimates the stiffness, and therefore the stress, within the tissue.

The advantages of using the Hooke model is related to the computational convenience in the solution of the elastic problem: the algorithm is rather fast and it has been possible to conduct numerous analyses considering twelve corneas. In this respect, it has been possible to observe that one of the key points in the procedure is the alignment of the corneas in order to allow the comparison. Specifically, images of the eye may be affected by some unavoidable change of reference system. Optical machines detect at best the NT direction, so that images are generally taken with a minimum difference in terms or rotation. However, misplacement in the optical axis directions is always possible. Thus, after creating of the solid model, it may be necessary to perform some additional relative roto-translation to minimize the geometrical differences. In our approach, we refer to the limbus geometry as the one that undergoes the minimum changes also after PRK surgery and conduct all sorts of re-alignment using the limbus as reference. The particular unsuccessful case of the cornea labelled 1D is exemplary in this regard. We believe that the images have been affected by some unwanted bias that we were not able to detect. Therefore, in general, it is important that the images are taken with the same tomographer operated by the same surgeon to minimize the differences.

The identified geometry and set of material properties obtained by the approach will be dependent also on the patient-specific IOP. In this study, we used an ideal IOP $(14 \mathrm{mmHg}$ ) because this information was not available. The fact that the IOP was not patient-specific can also justify the fact that some corneas showed clear optimal parameter values, whereas for others the procedure failed. It is important to note that, in general, IOP measured by tonometers is biased due to corneal stiffness. Many tonomoters are based on correction tables, often based on a wide set of numerical calculations conducted on ideal corneas to provide a less biased indication of IOP. This expedient does not avoid to obtain the patient-specific IOP, but only an averaged value. We believe that IOP has to be considered as an unknown of the problem, and it must be identified, together with the stress-free geometry and material properties, in a more sophisticated inverse analysis, which is presently the object of a study in our group.

The difficulties observed in using unrealistic materials (Hooke and MooneyRivlin) for the identification of an anisotropic body are indeed less marked when a more accurate material model is selected, as it has been observed in our previous applications. ${ }^{5,32,40}$ As matter of fact, the ML index plot is characterized by the presence of a well-identified minimum, with smaller values, in the anisotropic case. 
This indicates that an accurate material model is the basis of any good numerical simulation.

A final comment must be given on the search approach used in this study, conducted in a rather rough way by varying slightly one of the material parameters at time, under some assumptions not really supported by clinical evidence. Thus, criticism can be raised for the choice of keeping Poisson's coefficient constant in the Hooke material model case, or in conducting two disjoined searches on a single parameter for the Mooney-Rivlin model case, or in excluding from the search the fibril stiffness and rigidity parameters for the anisotropic material model.

We are convinced that the optimization of the ML index must be conducted with more solid search algorithms, that include not only all the relevant material properties, but also the IOP as unknown action. The multi-objective search can be based on faster algorithms, typically used in inverse analysis, such as the conjugate gradient and methods based on Pareto optimality criteria.

\section{References}

1. Maurice DM. The structure and transparency of the cornea. J Physiol. 1957;136(2): 263-286.

2. Meek KM, Blamires T, Elliot GF, Gyi TJ, Nave C. The organization of collagen fibrils in the human corneal stroma: a synchroton x-ray diffraction study. Curr Eye Res. 1987;6, 841-846.

3. Daxer A, Fratzl P. Collagen fibril orientation in the human corneal stroma and its implication in keratoconus. Invest Ophthalmol Vis Sci. 1997;38, 121-129.

4. Munnerlyn CR, Koons SJ, Marshall J. Photorefractive keratectomy: a technique for laser refractive surgery. J Cataract Refract Surg. 1988;14(1): 46-52.

5. Simonini I, Pandolfi A. Customized finite element modelling of the human cornea. PLoS One. 2015;10(6): e0130426.

6. Boschetti F, Triacca V, Spinelli L, Pandolfi A. Mechanical Characterization of Porcine Corneas. J Biomech Eng. 2012;134(3): 031003-1-9.

7. Pinsky PM, Heide D van der, Chernyak D. Computational modeling of mechanical anisotropy in the cornea and sclera. J Cataract Refract Surg. 2005;31(1): 136-145.

8. Pandolfi A, Manganiello F. A material model for the human cornea. Biomech Model Mechanobiol. 2006;5, 237-246.

9. Aghamohammadzadeh $\mathrm{H}$, Newton $\mathrm{RH}$, Meek KM. X-ray scattering used to map the preferred collagen orientation in the human cornea and limbus. Structure. 2004;12(2): 249-256.

10. Quantock AJ, Boote C, Young RD, Hayes S, Tanioka H, Kawasaki S, et al. Small-angle fibre diffraction studies of corneal matrix structure: a depth-profiled investigation of the human eye-bank cornea. J Appl Crystallogr. 2007;40(s1): s335-s340.

11. Wollensak G, Spörl E, Mazzotta C, Kalinski T, Sel S. Interlamellar cohesion after corneal crosslinking using riboflavin and ultraviolet A light. Br J Ophthalmol. 2011;95(6): 876-880.

12. Petsche SJ, Chernyak D, Martiz J, Levenston ME, Pinsky PM. Depth-dependent transverse shear properties of the human corneal stroma. Invest Ophthalmol Vis Sci. 2012;53, 873-880.

13. Meek KM, Boote $\mathrm{C}$. The use of $\mathrm{X}$-ray scattering techniques to quantify the orientation and distribution of collagen in the corneal stroma. Prog Retin Eye Res. 2009;28(5): 369-392.

14. Grytz R, Downs JC. A forward incremental prestressing method with application to inverse parameter estimations and eye-specific simulations of posterior scleral shells. Comput Methods Appl Mech Eng. 2013;16(7): 768-780. 
15. Raghavan ML, Ma B, Fillinger MF. Non-invasive determination of zero-pressure geometry of arterial aneurysms. Ann Biomed Eng. 2006;34(9): 1414-1419.

16. Bols J, Degroote J, Trachet B, Verhegghe B, Segers P, Vierendeels J. A computational method to assess the in vivo stresses and unloaded configuration of patient-specific blood vessels. J Comput Appl Math. 2013;246, 10-17.

17. Bols J, Degroote J, Trachet B, Verhegghe B, Segers P, Vierendeels J. Inverse modelling of image-based patient-specific blood vessels: zero-pressure geometry and in vivo stress incorporation. ESAIM: Math Model Num. 2013;47(4): 1059-1075.

18. Lu J, Zhou X, Raghavan ML. Inverse elastostatic stress analysis in pre-deformed biological structures: demonstration using abdominal aortic aneurysms. J Biomech. 2007;40(3): 693-696.

19. De Putter S, Wolters BJ, Rutten MC, Breeuwer M, Gerritsen FA, Vosse FN Van de. Patient-specific initial wall stress in abdominal aortic aneurysms with a backward incremental method. J Biomech. 2007;40(5): 1081-1090.

20. Gee MW, Reeps $\mathrm{CH}$, Eckstein $\mathrm{HH}$, Wall WA. Prestressing in finite deformation abdominal aortic aneurysm simulation. J Biomech. 2009;42(11): 1732-1739.

21. Pandolfi A, Holzapfel GA. Three-dimensional modelling and computational analysis of the human cornea considering distributed collagen fiber orientation. J Biomech Eng. 2008;130, 061006.

22. Pandolfi A, Fotia G, Manganiello F. Finite element simulations of laser refractive corneal surgery. Eng Comput. 2009;25(1): 15-24.

23. Pandolfi A, Vasta M. Fiber distributed hyperelastic modeling of biological tissues. Mech Mat. 2012;44, 151162.

24. Ariza-Gracia MA, Zurita JF, Piñero DP, Rodriguez-Matas JF, Calvo B. Coupled biomechanical response of the cornea assessed by non-contact tonometry. A simulation study. PLoS One. 2015;10(3): e0121486.

25. Ariza-Gracia MÁ, Zurita J, Piñero DP, Calvo B, Rodriguez-Matas JF. Automatized patient-specific methodology for numerical determination of biomechanical corneal response. Ann Biomed Eng. 2016;44(5): 1753-1772.

26. Ariza-Gracia MÁ, Redondo S, Llorens DP, Calvo B, Rodriguez-Matas JF. A predictive tool for determining patient-specific mechanical properties of human corneal tissue. Comput Methods Appl Mech Eng. 2017;317, 226-247.

27. Ariza-Gracia MÁ, Wu W, Calvo $B$, Malvè $M$, Büchler $P$, Rodriguez-Matas JF. Fluid-structure simulation of a general non-contact tonometry. A required complexity? Comput Methods Appl Mech Eng. 2018;

28. Otani T, Tanaka M. Unloaded shape identification of human cornea by variational shape optimization. Comput Methods Biomech Biomed Engin. 2018; 1-8.

29. Wollensak G, Spöerl E, Seiler T. Stress-strain measurements of human and porcine corneas after riboflavin-ultraviolet-A-induced cross-linking. J Cataract Refract Surg. 2003;29, 1780-1785.

30. Elsheikh A. Finite Element Modeling of Corneal Biomechanical Behavior. J Refract Surg. 2010;26(4): 289300.

31. Seven I, Vahdati A, De Stefano VS, Krueger RR, Dupps WJ. Comparison of patient-specific computational modeling predictions and clinical outcomes of LASIK for myopia. Invest Ophthalmol Vis Sci. 2016;57(14): 6287-6297.

32. Montanino A, Gizzi A, Vasta M, Angelillo M, Pandolfi A. Modeling the biomechanics of the human cornea accounting for local variations of the collagen fibril architecture. J Appl Math Mech. 2018;98(12): 21222134.

33. Studer H, Riedwyl H, Büchler P. Importance of multiple loading scenarios for the identification of material coefficients of the human cornea. Comput Methods Appl Mech Eng. 2012;15(1): 93-99.

34. Kok S, Botha N, Inglis HM. Calibrating corneal material model parameters using only inflation data: An ill-posed problem. Int J Numer Method Biomed Eng. 2014;30(12): 1460-1475.

35. Petsche SJ, Pinsky PM. The role of 3-D collagen organization in stromal elasticity: a model based on Xray diffraction data and second harmonic-generated images. Biomech Model Mechan. 2013;12(6): 11011113. 
36. Simonini I, Pandolfi A. The influence of intraocular pressure and air jet pressure on corneal contactless tonometry tests. J Mech Behav Biomed Mater. 2016;58, 75-89.

37. Simonini I, Angelillo M, Pandolfi A. Theoretical and numerical analysis of the corneal air puff test. J Mech Phys Solids. 2016;93, 118-134.

38. Montanino A, Angelillo M, Pandolfi A. Modelling with a meshfree approach the cornea-aqueous humor interaction during the air puff test. J Appl Math Mech. 2018;77, 205-216.

39. Montanino A, Angelillo M, Pandolfi A. A 3D fluid-structure interaction model of the air puff test in the human cornea. J Mech Behav Biomed Mater. 2019;94, 22-31.

40. Sánchez $P$, Moutsouris K, Pandolfi A. Biomechanical and optical behavior of human corneas before and after photorefractive keratectomy. J Cataract Refract Surg. 2014;40(6): 905-917. 